(2) Open Access Full Text Article

REVIEW

\title{
Assessment of the acute abdomen: role of the plain abdominal radiograph
}

\author{
Frances A Hampson \\ Ashley S Shaw \\ Department of Radiology, \\ Addenbrooke's Hospital, \\ Cambridge, UK
}

\author{
This article was published in the following Dove Press journal: \\ Reports in Medical Imaging \\ I5 November 2010 \\ Number of times this article has been viewed
}

Correspondence: Frances A Hampson Department of Radiology, Addenbrooke's Hospital, Hills Road, Cambridge, CB2 0QQ, UK

Tel +447967646472

Email frances.hampson@addenbrookes. nhs.uk

\begin{abstract}
The acute abdomen' is one of the most frequent reasons for presentation to the emergency department. There are a multitude of potential causes. Imaging plays a vital role in making an accurate and timely diagnosis, which in turn reduces morbidity and mortality. Plain abdominal radiography has traditionally been considered a fundamental part of the initial assessment of acute abdominal pain. However, with the increasing availability of computed tomography, ultrasound, and magnetic resonance imaging, the role of the plain abdominal radiograph has been increasingly questioned and its use is dwindling. During the course of this review, we will describe the current guidelines for the use of the plain abdominal radiograph and assess its potential diagnostic yield. We will also discuss its current applications in the context of the acute abdomen both in general and in a number of frequently encountered conditions and illustrate when other imaging modalities would be better employed.
\end{abstract}

Keywords: acute abdomen, abdominal radiograph

\section{Introduction}

Sudden onset of severe abdominal pain that requires emergency treatment, better known as 'the acute abdomen,' is one of the most frequent reasons for presentation at the emergency department (ED).

There are a multitude of potential causes. A review of 10,682 patients presenting with an acute abdomen found that $28 \%$ had appendicitis, $9.7 \%$ cholecystitis, $4.1 \%$ small bowel obstruction (SBO), 4\% a gynecological disorder, 2.9\% pancreatitis, $2.9 \%$ renal colic, $2.5 \%$ peptic ulcer disease, $1.5 \%$ cancer, $1.5 \%$ diverticular disease, and $9 \%$ had a variety of less common conditions. ${ }^{1}$ One-third of patients were not given a definite diagnosis.

Modern imaging techniques play a vital role in the investigation of the acute abdomen. An accurate and timely diagnosis is needed to minimize morbidity and mortality. Given the relatively nonspecific nature of both physical examination and laboratory investigations, there is a consensus that patients should be imaged at an early stage..$^{2-5}$

Plain abdominal radiography has traditionally been considered a fundamental part of the initial assessment of the acute abdomen. As such, it is often the first radiological investigation in the ED. However, with the increasing availability of computed tomography (CT), ultrasound, and magnetic resonance imaging (MRI), the role of the 
plain abdominal radiograph has been increasingly questioned and its use is dwindling.

CT can provide a comprehensive review of the abdomen and pelvis. It is now widely considered the imaging technique of choice for the majority of patients presenting with an acute abdomen. ${ }^{6-10}$ The limitations of plain film radiography and ultrasound, together with the advent of multidetector CT (MDCT), have facilitated this. MDCT systems have enabled greater volume coverage and thinner slice acquisition without significant increases in radiation burden. ${ }^{7,9}$ Ultrasound retains some advantages over $\mathrm{CT}$, such as the absence of ionizing radiation, and is the investigation of choice in certain situations.

The potential of MRI for investigating certain causes of acute abdominal pain is currently being explored. Despite the long imaging times, high costs, and limited availability of MRI, the absence of ionizing radiation makes it an appropriate tool in the investigation of selected patients, particularly pregnant women and young patients, who have undergone a nondiagnostic or equivocal ultrasound examination. MRI can, for instance, be very useful in the assessment of pregnant patients with lower abdominal pain that is believed to have an extrauterine etiology, such as ovarian torsion or appendicitis.

During the course of this review, we will describe the current guidelines for the use of the plain abdominal radiograph and assess its potential diagnostic yield. We will also discuss its current applications in the context of the acute abdomen both in general and in a number of frequently encountered conditions and illustrate when other imaging modalities would be better employed.

\section{Current guidelines for the use of plain abdominal radiographs in the assessment of the acute abdomen}

The Royal College of Radiologists (RCR) has published guidelines for the use of plain abdominal radiographs. ${ }^{11}$ They are summarized in Table 1 .

The American College of Radiology (ACR) revised their guidelines for the use of the plain abdominal radiograph in 2006. ${ }^{12}$ The indications are listed in Table 2. They state that there are no absolute contraindications to abdominal radiography. They also state that in many clinical situations, other imaging modalities may be more appropriate as the initial or only examination for a patient with abdominal disease.
Table I Summary of the RCR guidelines for the use of the plain abdominal radiograph

\begin{tabular}{ll}
\hline $\begin{array}{l}\text { Plain abdominal } \\
\text { radiograph indicated }\end{array}$ & $\begin{array}{l}\text { Plain abdominal } \\
\text { radiograph only indicated } \\
\text { in specific circumstances }\end{array}$ \\
\hline - Acute abdominal pain with & - Palpable mass \\
suspected perforation/obstruction & \\
- Acute small bowel obstruction: & - Constipation \\
confirmation and assessment of level & \\
- Acute large bowel & - Suspected ureteric \\
obstruction & colic/calculi \\
- Acute exacerbation of & - Foreign body in \\
inflammatory bowel disease & pharynx/upper esophagus \\
- Acute abdominal pain & Smooth and small foreign \\
warranting hospital admission & body, eg, a coin \\
for consideration of surgery & \\
- Acute pancreatitis: may & \\
enable exclusion of perforation & \\
or intestinal obstruction & \\
as alternative cause of pain & \\
- Chronic pancreatitis: may & \\
show calcifications & \\
- Renal failure & \\
- Hematuria & \\
- Sharp/poisonous foreign body & \\
- Blunt or stab abdominal injury & \\
\hline
\end{tabular}

\section{The potential diagnostic yield of the plain abdominal radiograph}

The fact that the abdominal radiograph is still considered to have so many potential indications is somewhat surprising. There is little evidence to support such widespread usage of the plain abdominal radiograph in patients with acute abdominal pain. ${ }^{13}$ It is no secret that plain abdominal radiographs are often nonspecific and are easily misinterpreted. Interpretation is prone to interobserver variability, particularly where certain findings are concerned. One study showed excellent interobserver agreement $(\kappa$-value $>0.75)$ for pneumobilia, renal calculi, and pneumoperitoneum, while there was fair to good agreement ( $\kappa$-value $0.40-0.75$ ) for SBO, gallstones, colitis, thumbprinting, dilated loops of bowel, abnormal air-fluid levels, normal gas pattern, and soft tissue mass. There was poor agreement $(\kappa$-value $<0.40)$ for large bowel obstruction (LBO), nonspecific bowel gas pattern, completeness of SBO, location of SBO, generalized ileus, localized ileus, ascites, and ureteric calculi. ${ }^{14}$

Four recent studies have illustrated the limited diagnostic yield of the plain abdominal radiograph. Ahn et al ${ }^{15}$ retrospectively reviewed the records of 1000 consecutive adult patients presenting to the ED. Eight hundred and seventy-one patients underwent plain abdominal radiography, and 188 
Table 2 The ACR guidelines for the use of the plain abdominal radiograph indications

- Abdominal, flank, or pelvic pain

- Vomiting

- Abdominal distension, bloating, or increased girth

- Evaluation for and follow-up of bowel obstruction or nonobstructive ileus

- Constipation

- Diarrhea

- Evaluation of necrotizing enterocolitis in the premature newborn

- Palpable abdominal mass or organomegaly

- Evaluation of congenital abnormalities

- Follow-up of the postoperative patient

- Evaluation for pneumoperitoneum

- Follow-up to contrast

examinations of the gastrointestinal

or urinary tract

underwent abdominal CT. Interpretation of the abdominal radiographs was nonspecific in $68 \%$ of cases, normal in $23 \%$, and abnormal in $10 \%$. Abdominal radiography had $0 \%$ specificity for appendicitis, pyelonephritis, pancreatitis, and diverticulitis. This is not surprising given that these common conditions do not have any specific radiographic features. The highest sensitivities of abdominal radiography were $90 \%$ for intra-abdominal foreign body and $49 \%$ for bowel obstruction. They therefore found that the diagnostic yield of the abdominal radiograph in this group of patients was low. This to some extent was due to the fact that $68 \%$ of the interpretations were nonspecific and hence by definition could not be diagnostic.

MacKersie et al ${ }^{16}$ prospectively evaluated and compared the diagnostic accuracy of unenhanced CT for patients presenting with a nontraumatic acute abdomen with that of traditional abdominal radiography. Ninetyone adult patients were examined over a 7-month period. The patients underwent a three-view acute abdominal radiograph series (an erect chest radiograph and erect and supine abdominal radiographs) and an unenhanced helical CT. Unenhanced helical CT was found to have an overall sensitivity, specificity, and accuracy of $96.0 \%, 95.1 \%$, and $95.6 \%$, respectively. The acute abdominal radiograph series was found to have an overall sensitivity, specificity, and accuracy of $30.0 \%, 87.8 \%$, and $56 \%$, respectively. Both Ahn et al and MacKersie et al concluded that abdominal radiographs are insensitive in the evaluation of adult patients with nontraumatic acute abdominal pain.

Kellow et $\mathrm{al}^{17}$ retrospectively reviewed the interpretations of the initial plain abdominal radiographs of 874 patients who presented to the ED with acute nontraumatic abdominal pain. The patients' medical records were also reviewed to determine whether further imaging was performed. If further imaging had been performed, the results were compared with abdominal radiography. Interpretation of abdominal radiography was normal in 34\%, nonspecific in $46 \%$, and abnormal in $19 \%$. Additional imaging was requested in $42 \%$ of cases with normal radiography results, $52 \%$ of cases with nonspecific results, and $59 \%$ of cases with abnormal results. Of those with normal abdominal radiography, $72 \%$ were found to have abnormal findings at follow-up imaging. This figure increased to $78 \%$ for nonspecific abdominal radiography results and $87 \%$ for abnormal results. Abdominal radiography helped confirm the suspected diagnosis in only $2 \%-8 \%$ for all indications other than catheter placement. Initial abdominal radiography was perhaps helpful in changing patient management without further imaging in only $4 \%$ of cases, demonstrating that plain abdominal radiography rarely contributes to patient management.

Most recently, van Randen et $\mathrm{al}^{18}$ conducted a multicenter prospective study entitled 'The role of plain radiographs in patients with acute abdominal pain at the ED' on behalf of the optimization of diagnostic imaging use in patients with acute abdominal pain (OPTIMA) study group. The aim of the OPTIMA study group is to provide an evidence base for constructing optimal diagnostic imaging guidelines for patients with acute abdominal pain presenting to the ED. The aim of the recent study was to evaluate the added value of plain radiographs (supine abdominal and erect chest radiographs) on top of clinical assessment in patients presenting to the ED with acute abdominal pain. The diagnosis assigned by the treating physician changed after plain radiography in only 117 out of 1021 cases. This change was only correct in 39 cases. The clinical diagnosis was correct in $49 \%$ of cases. The diagnosis after evaluation of the plain radiographs was correct in $50 \%$ of cases, which is an insignificant difference. Only sensitivity in detecting bowel obstruction was significantly higher after evaluation of the plain radiographs. Furthermore, in the $65 \%$ of cases with unaltered diagnosis before and after plain abdominal radiography, the level of confidence of that diagnosis did not change. The group concluded that the added value of plain radio- 

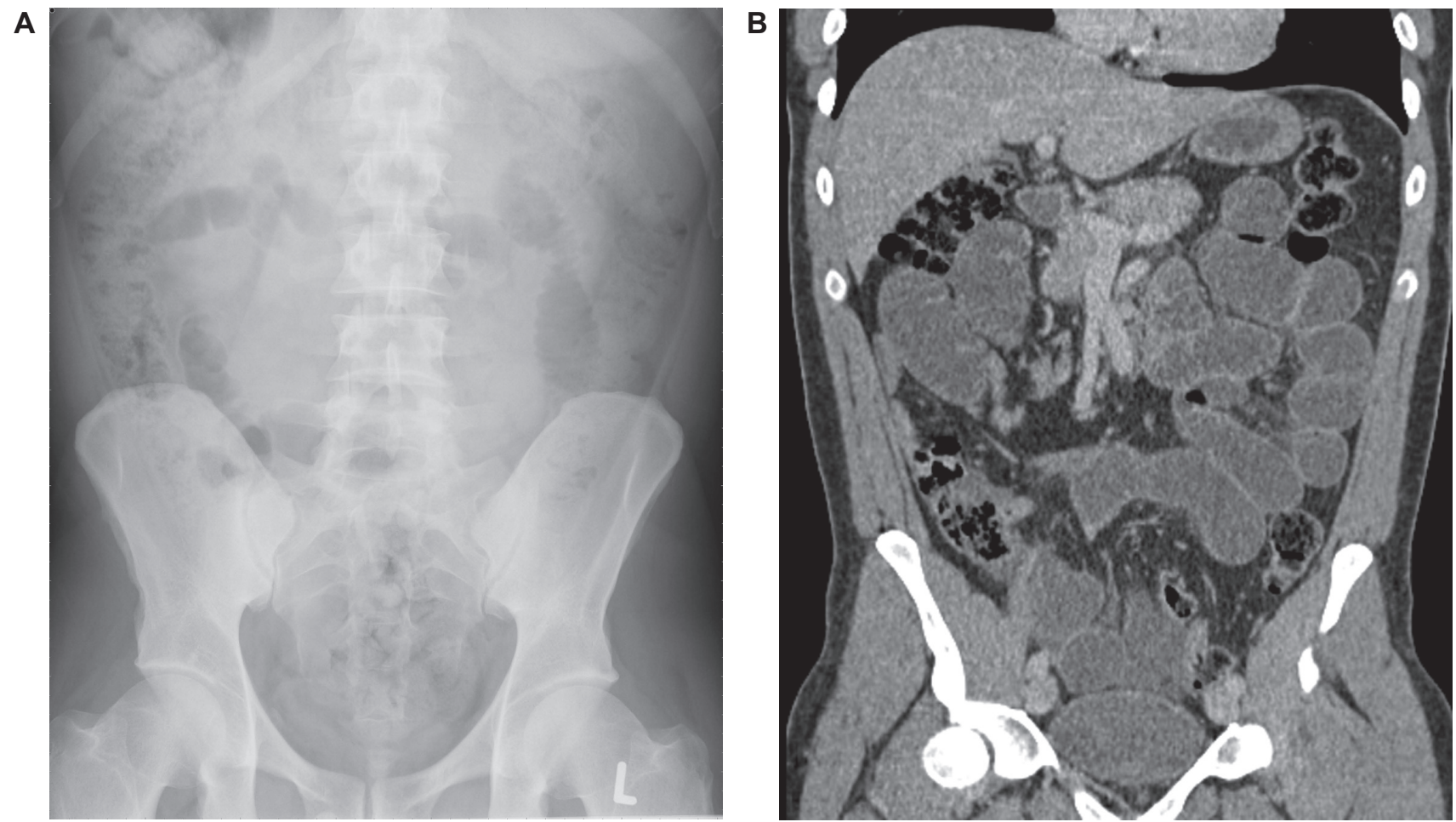

C

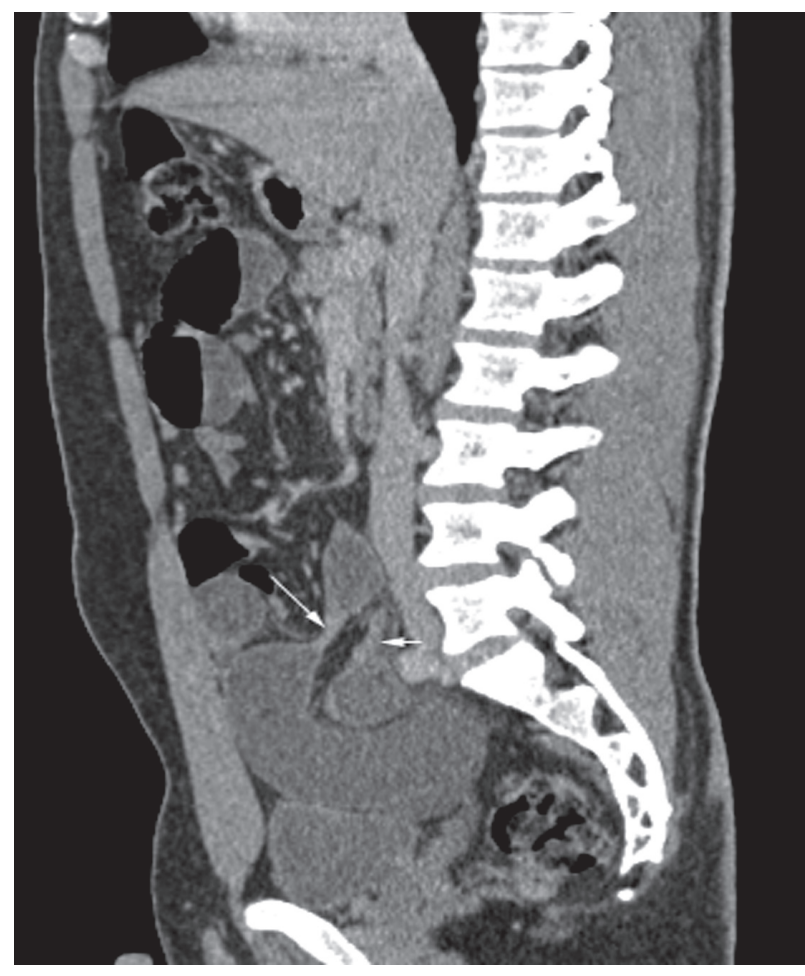

D

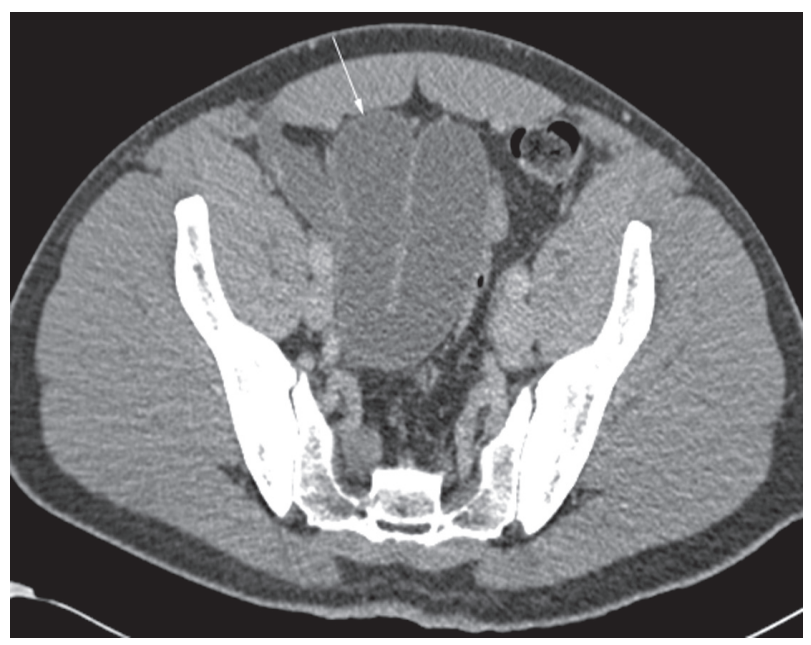

Figure I Thirty-two-year-old man presenting with vomiting, abdominal pain, and guarding postappendectomy. A) Plain abdominal radiograph showing a solitary dilated loop of small bowel in the left side of the abdomen and no convincing features of obstruction. B) Coronal reformat of a subsequent CT examination acquired following the administration of intravenous contrast medium demonstrated multiple dilated loops of fluid-filled small bowel. C) Sagittal reformat of the same CT examination demonstrating an obstructed closed loop. The transition points are indicated by the white arrows. D) Axial CT image demonstrating the closed loop (white arrow). 

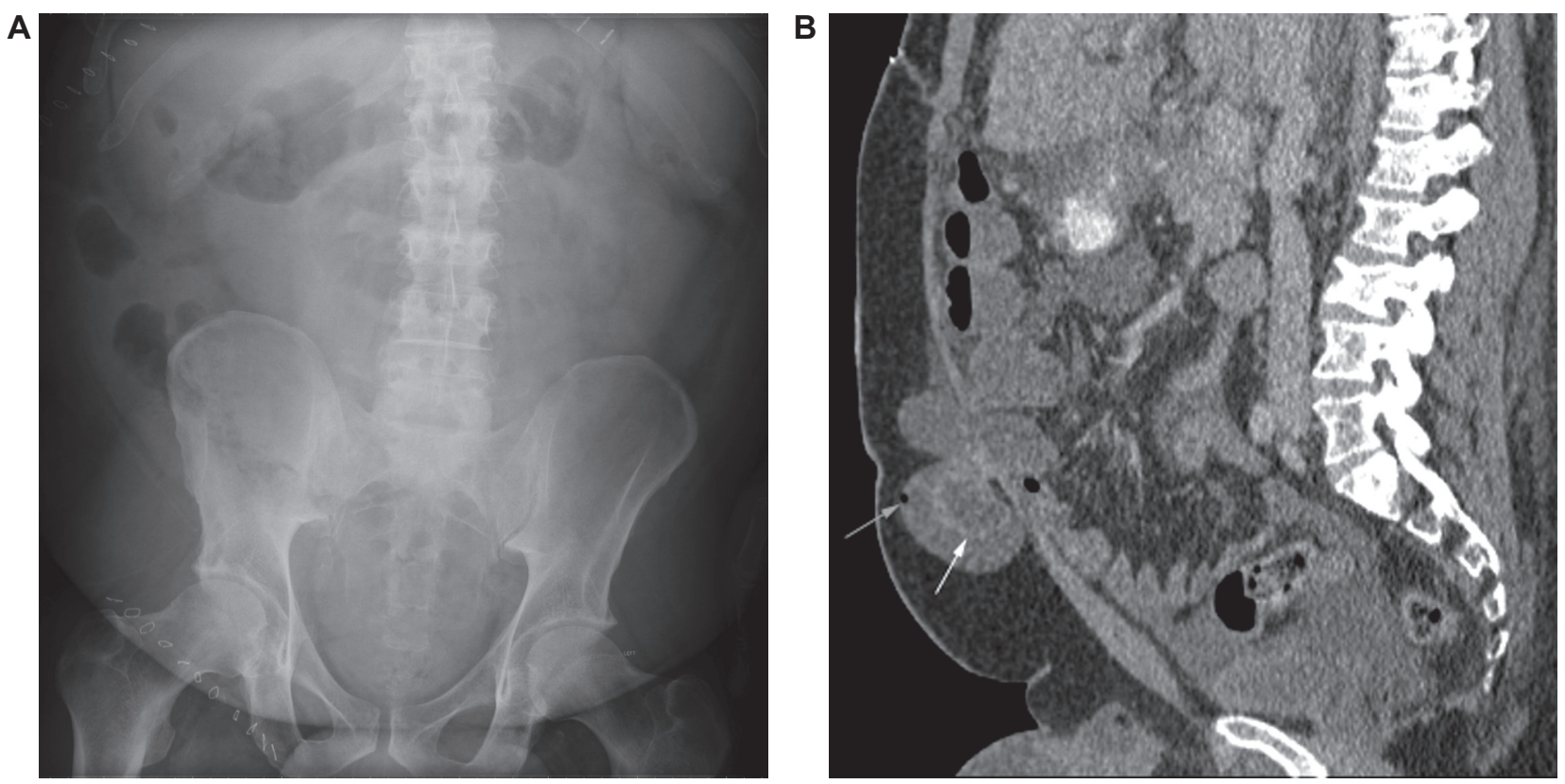

Figure 2 Fifty-one-year-old man presenting with abdominal pain and vomiting 4 days post liver transplant and right inguinal hernia repair. A) Plain abdominal radiograph demonstrating a prominent loop of small bowel in the center of the abdomen. No definite features to suggest obstruction. B) Sagittal reformat of a CT examination acquired following the administration of intravenous contrast medium demonstrating high-grade SBO. The transition point is within a paraumbilical hernia (white arrow). There is adjacent free fluid and a locule of free gas (grey arrow) indicating perforation.

graphs is too limited to advocate their routine use in the diagnostic workup of patients with acute abdominal pain. They went on to suggest that plain radiography should be excluded from the initial assessment of patients with acute abdominal pain presenting to the ED.

\section{The role of the plain abdominal radiograph in the diagnosis of specific causes of acute abdominal pain}

The recent studies therefore do not support the widespread use of the plain abdominal radiograph in the assessment of the acute abdomen. We will now review some common causes of acute abdominal pain and discuss the role of the plain abdominal radiograph in direct relation to them.

\section{Bowel obstruction}

SBO is a relatively common cause of acute abdominal pain. The most common causes of SBO are adhesions and hernias. Clinical evaluation has limitations in the diagnosis of SBO. The combination of vomiting, increased bowel sounds, and a distended abdomen has a positive predictive value for SBO of $64 \% .{ }^{19}$ Imaging is therefore utilized at an early stage. If patients are to be managed appropriately, it is crucial to identify the cause of obstruction and to determine whether the obstruction is partial or complete. Furthermore, it is essential to identify closed-loop obstructions (Figure 1) at an early stage in order to expedite surgical intervention and avoid ischemia.

Plain abdominal radiography has traditionally been the primary imaging technique for patients with suspected SBO. It has been reported to have $46 \%-90.8 \%{ }^{15,19-22}$ sensitivity and a specificity of around $50 \%{ }^{20,21}$ Frager et al ${ }^{22}$ found that the combination of clinical findings and the interpretation of the plain abdominal radiographs led to a definitive diagnosis of SBO in only $46 \%$ of cases. Furthermore, this approach only had a sensitivity of $30 \%$ for the detection of partial SBO.

$\mathrm{CT}$ is a much more accurate technique for the diagnosis of SBO. It has a reported sensitivity of $93 \%$ and a specificity of $100 \%{ }^{20}$ The sensitivity of CT is significantly reduced for the diagnosis of partial SBO (64\%) as opposed to complete $\mathrm{SBO},{ }^{21}$ but it is still much more sensitive than the plain abdominal radiograph. Furthermore, the plain abdominal radiograph cannot be relied upon to supply the exact location or cause of SBO. ${ }^{22}$ Indeed, one of the main advantages of CT is its ability to diagnose the cause of obstruction, which in turn guides management (Figures 2 and 3 ).

Some authors advocate that clinicians should continue to use the plain abdominal radiograph as the initial imaging technique in the evaluation of patients with SBO whilst maintaining a low threshold for $\mathrm{CT}$ if there is a high clinical suspicion. ${ }^{13}$ Given that the diagnosis can be so frequently 

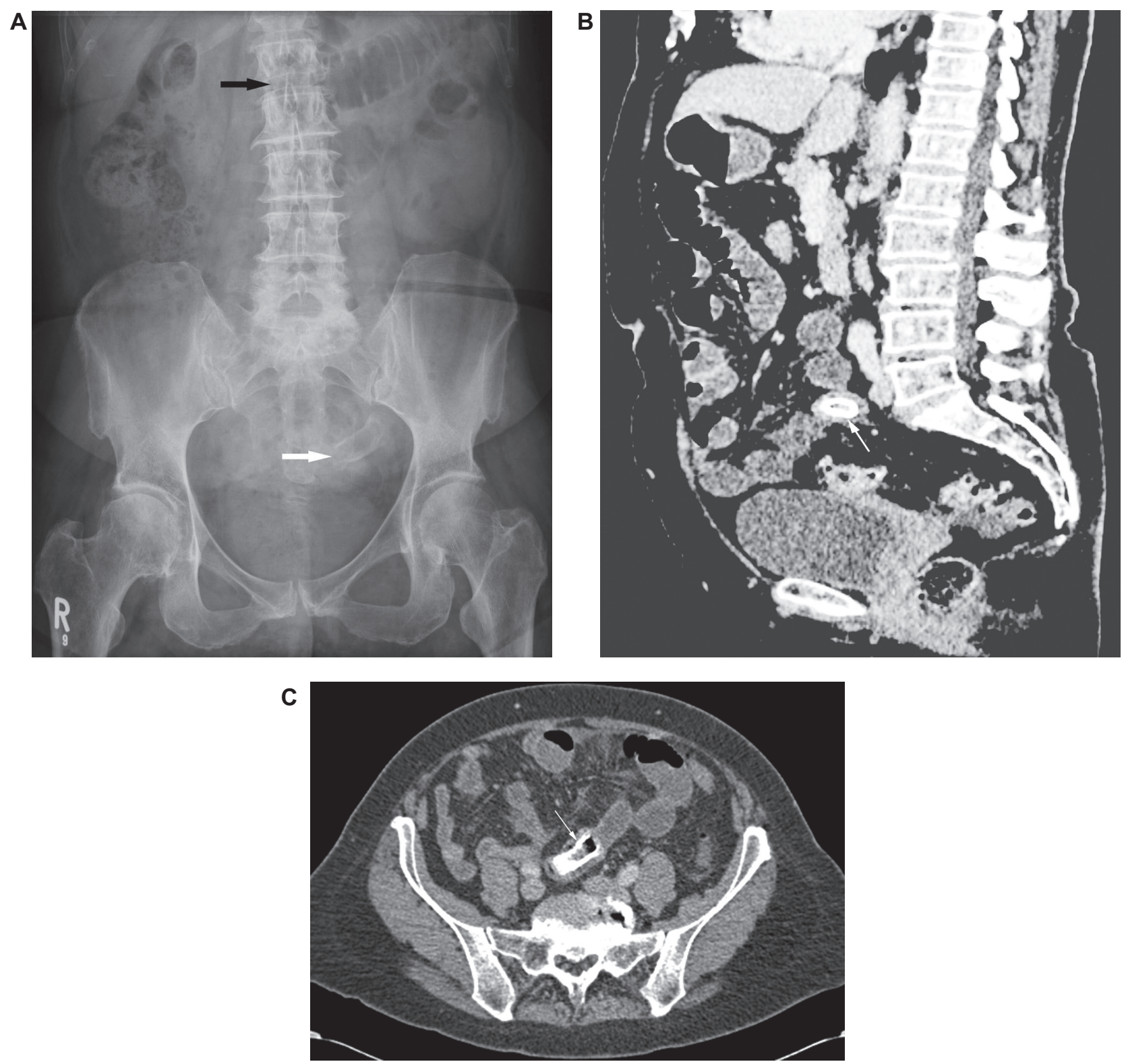

Figure 3 Seventy-four-year-old woman presenting with abdominal pain, vomiting, diarrhea, and weight loss. A) Plain abdominal radiograph demonstrating a dilated loop of small bowel in the right upper abdomen (black arrow). There is also a partially calcified opacity in the left hemi pelvis (white arrow). B) and C) Axial image and a sagittal reformat of a CT examination acquired following the administration of intravenous contrast medium demonstrating SBO caused by a partially calcified ingested foreign body (white arrow)

missed with plain radiography, this strategy is questionable. The ACR suggests that CT with intravenous contrast medium is the most appropriate test when complete or high-grade obstruction is suspected. If low-grade or intermittent SBO is suspected, several CT techniques (using barium or water as the contrast agent, enteroclysis) or small bowel examinations (follow-through or enteroclysis) are appropriate. ${ }^{23}$ Overall, we believe that $\mathrm{CT}$ should be considered the primary imaging modality for suspected SBO.
Traditionally, plain abdominal radiograph has been the primary imaging technique used to assess suspected cases of LBO. CT is generally used to demonstrate the level and cause of obstruction and identify any associated complications such as perforation (Figure 4). We believe that CT should be the primary imaging technique of choice in patients with suspected LBO as the plain abdominal radiograph may be inconclusive and confusing and hence lead to a delay in further management. ${ }^{24}$ 

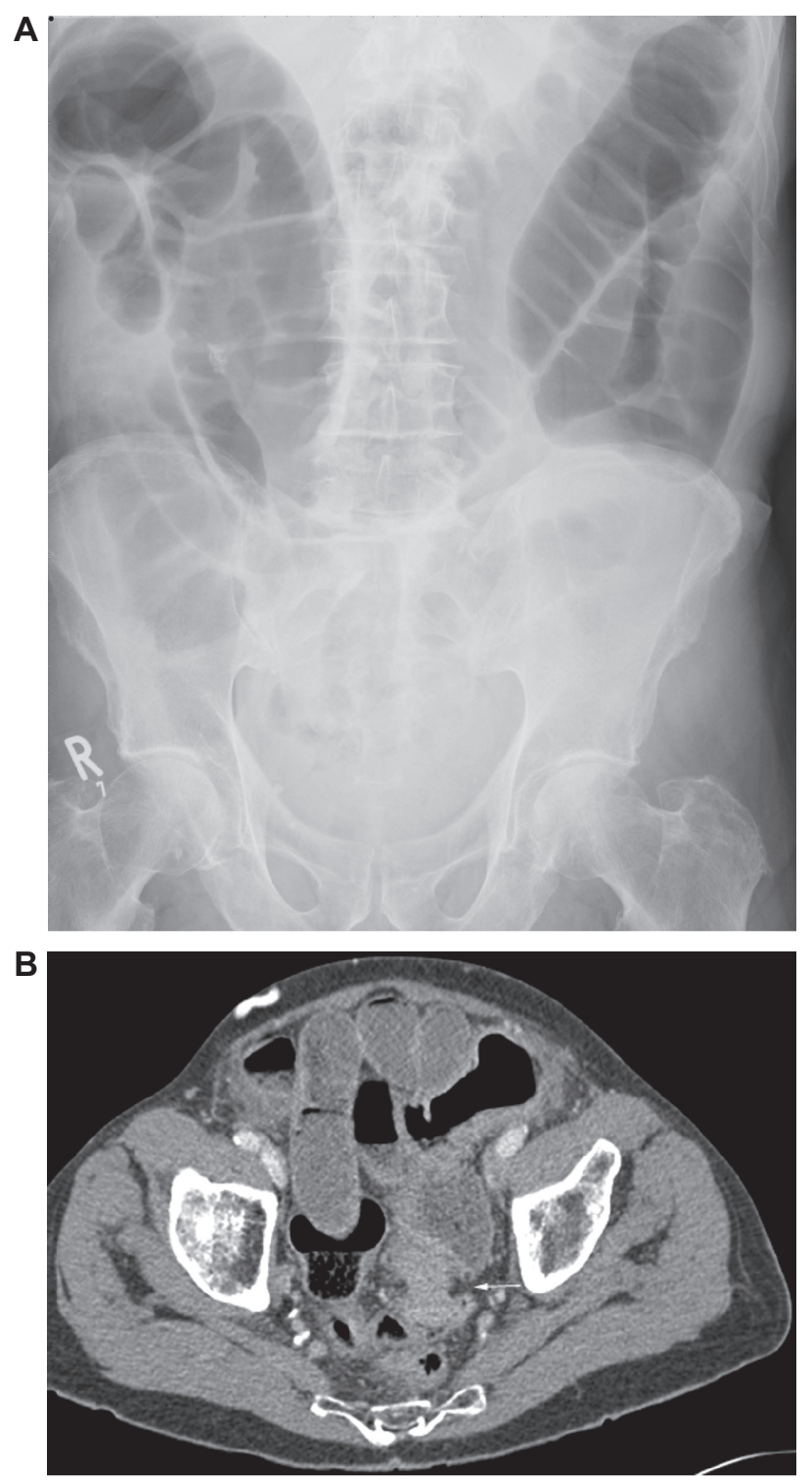

Figure 4 Eighty-five-year-old man presenting with a 2-week history of constipation, abdominal distension, and pain. A) Plain abdominal radiograph demonstrating LBO. The point of obstruction appears to be in the descending colon. B) Axial CT image acquired following the administration of intravenous contrast medium demonstrating an obstructing tumor with an apple core configuration (white arrow) in the sigmoid colon.

\section{Intra-abdominal foreign body}

Ingested foreign bodies are a common cause of presentation to the ED, particularly in the pediatric population. They are a potential cause of acute abdominal pain. Ahn et al ${ }^{15}$ reported that plain abdominal radiography had a sensitivity and specificity of $90 \%$ and $100 \%$, respectively, for the detection of intra-abdominal foreign bodies (Figure 5). The plain abdominal radiograph may therefore continue to have a role in this situation. Radiography should, however, only be used when demonstration of the foreign body is of

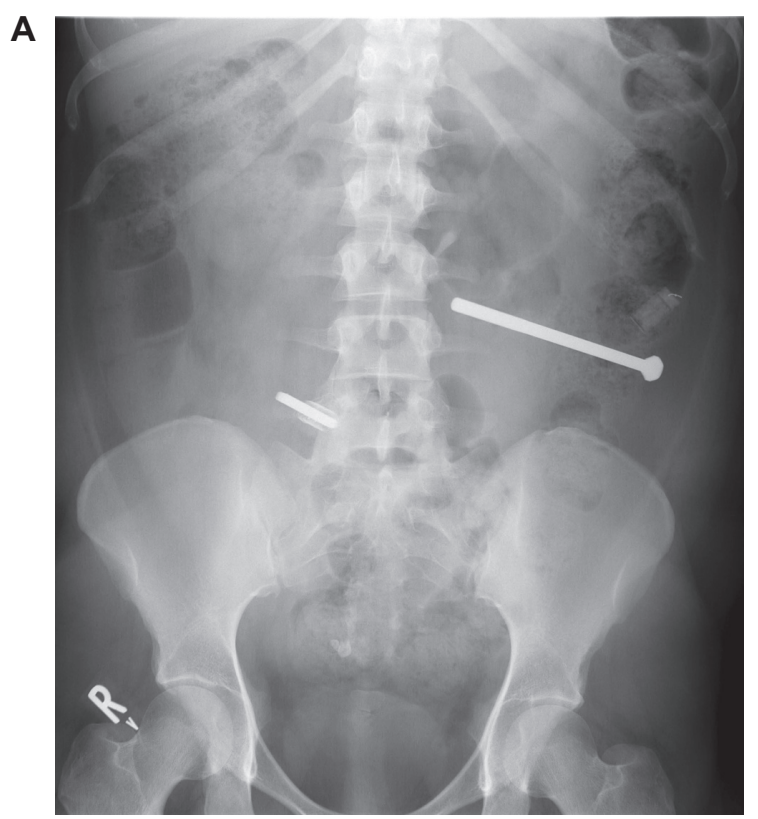

B

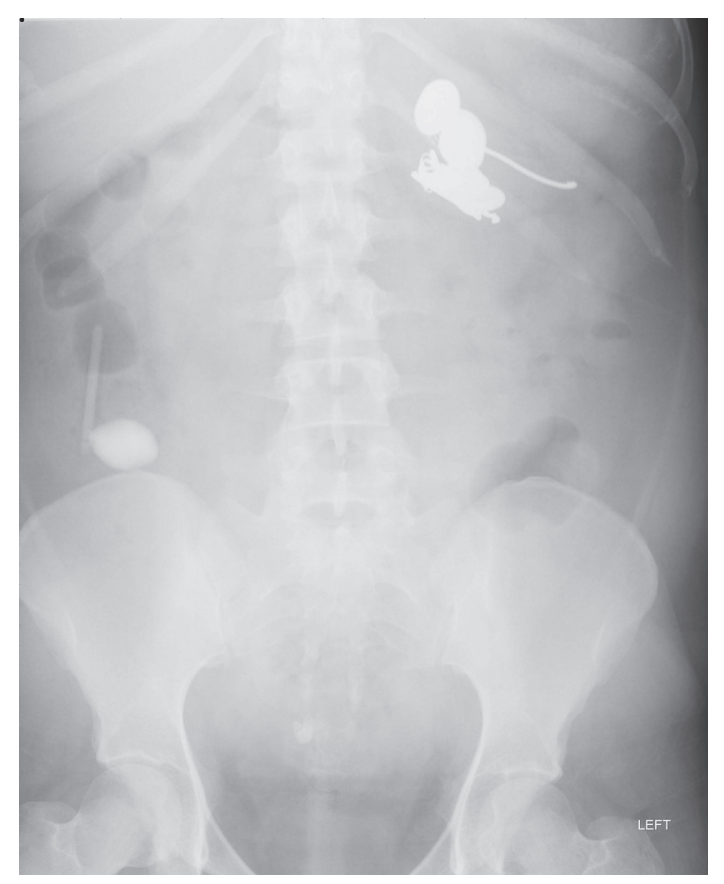

Figure 5 Twenty-three-year-old woman with psychiatric history. A) Plain abdominal radiograph demonstrating two ingested batteries and an ingested bolt. B) Followup plain abdominal radiograph demonstrating that the previously visualized foreign bodies had passed but several more had been ingested.

clinical relevance, that is, when it is potentially dangerous, for example, a battery (Figure 6), or when the patient is symptomatic.

\section{Renal calculi}

A number of studies have shown that unenhanced helical CT has a high sensitivity and specificity ${ }^{25-28}$ in the detection of ureteric calculi. It also allows alternative diagnoses to be 


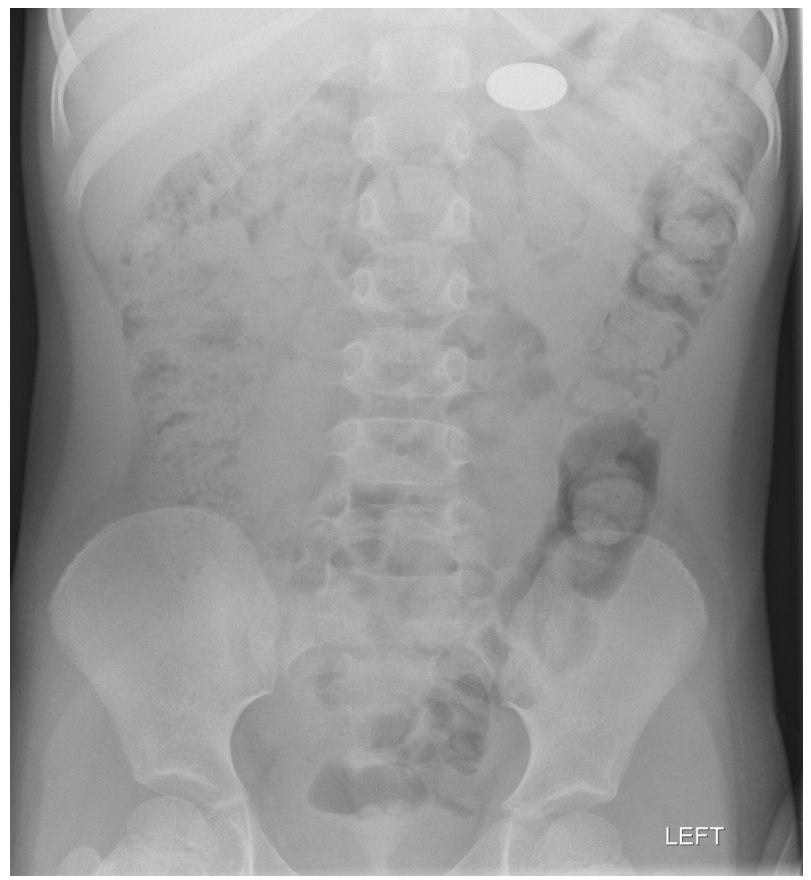

Figure 6 Plain abdominal radiograph of a 5-year-old boy who had ingested a coin battery. The battery is projected over the stomach.

made. ${ }^{28}$ As a result, it has replaced the intravenous urogram as the investigation of choice in the investigation of patients with suspected renal colic. Using CT as the gold standard, Levine et $\mathrm{al}^{29}$ found that the plain abdominal radiograph had a sensitivity of $45 \%$ and a specificity of $77 \%$ for the detection of ureteric calculi. There is therefore no need to perform a plain abdominal radiograph prior to $\mathrm{CT}$. Although plain abdominal radiographs may continue to have a part to play in the follow-up of renal calculi, they have no role to play in making the primary diagnosis (Figure 7).

\section{Acute appendicitis}

There is good evidence that plain abdominal radiography has no diagnostic value in patients suspected of having acute appendicitis. ${ }^{15,30} \mathrm{We}$ and many others therefore believe that there is no role for the plain radiograph in the investigation of patients with suspected appendicitis. Despite this, 50\%-75\% of patients with suspected appendicitis undergo plain radiography. ${ }^{30,31}$ Both ultrasound and CT have significant roles to play in the diagnosis of acute appendicitis. CT is more accurate $^{32}$ and is the generally preferred imaging technique (Figure 8). Ultrasound is used as the primary imaging technique in children and women of childbearing age. MRI may be helpful in the diagnosis of acute appendicitis in pregnant patients who have an inconclusive ultrasound examination. ${ }^{33-35}$
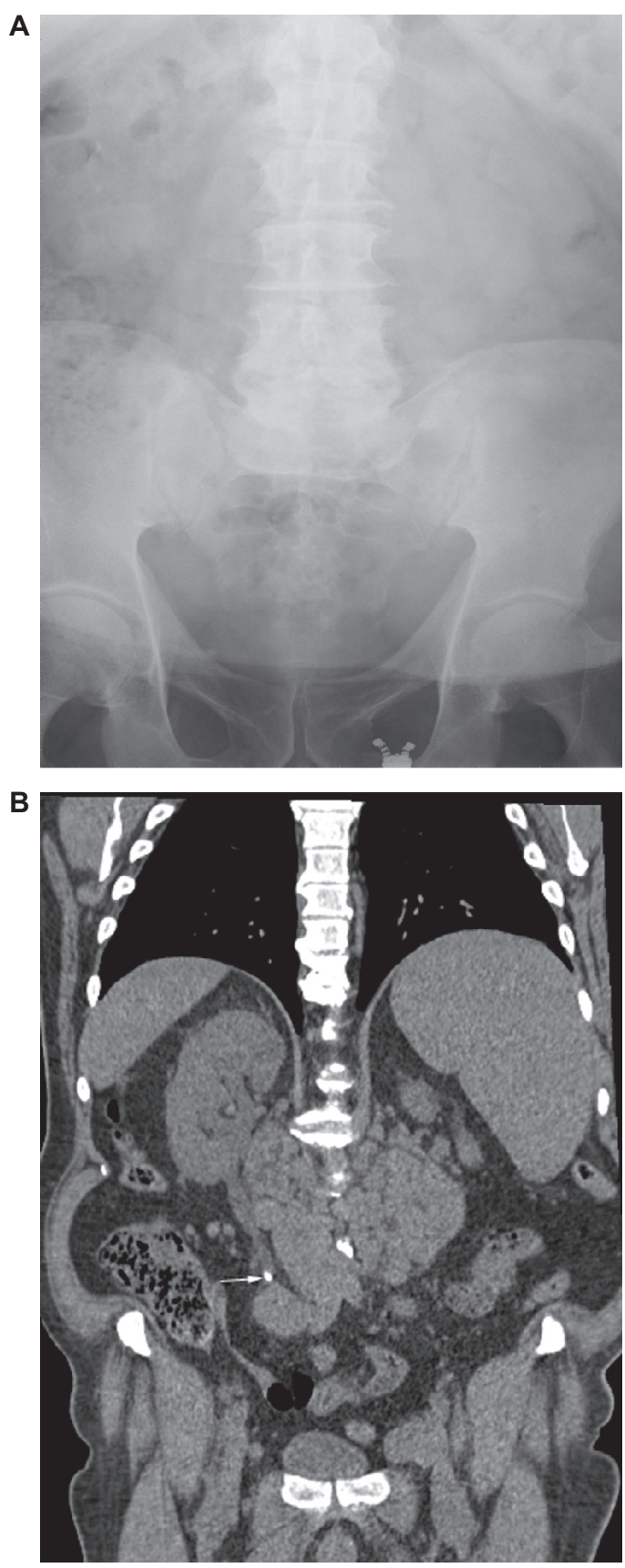

Figure 7 Sixty-year-old man with a history of chronic lymphocytic leukemia presenting with right flank pain. A) Plain abdominal radiograph demonstrating a small calculus projected over the lower pole of the right kidney but no definite ureteric calculi. B) Sagittal reformat of an unenhanced CT examination demonstrating a calculus within the right ureter (white arrow). There is associated mild pelvicalyceal and ureteric dilation. Retroperitoneal lymphadenopathy and splenomegaly also demonstrated.

\section{Acute diverticulitis, pancreatitis, and cholecystitis}

Acute colonic diverticulitis does not have any specific radiographic features. Plain abdominal radiographs are therefore unhelpful. CT is used to confirm the suspected diagnosis and detect potential complications. A sentinel loop or the colon 


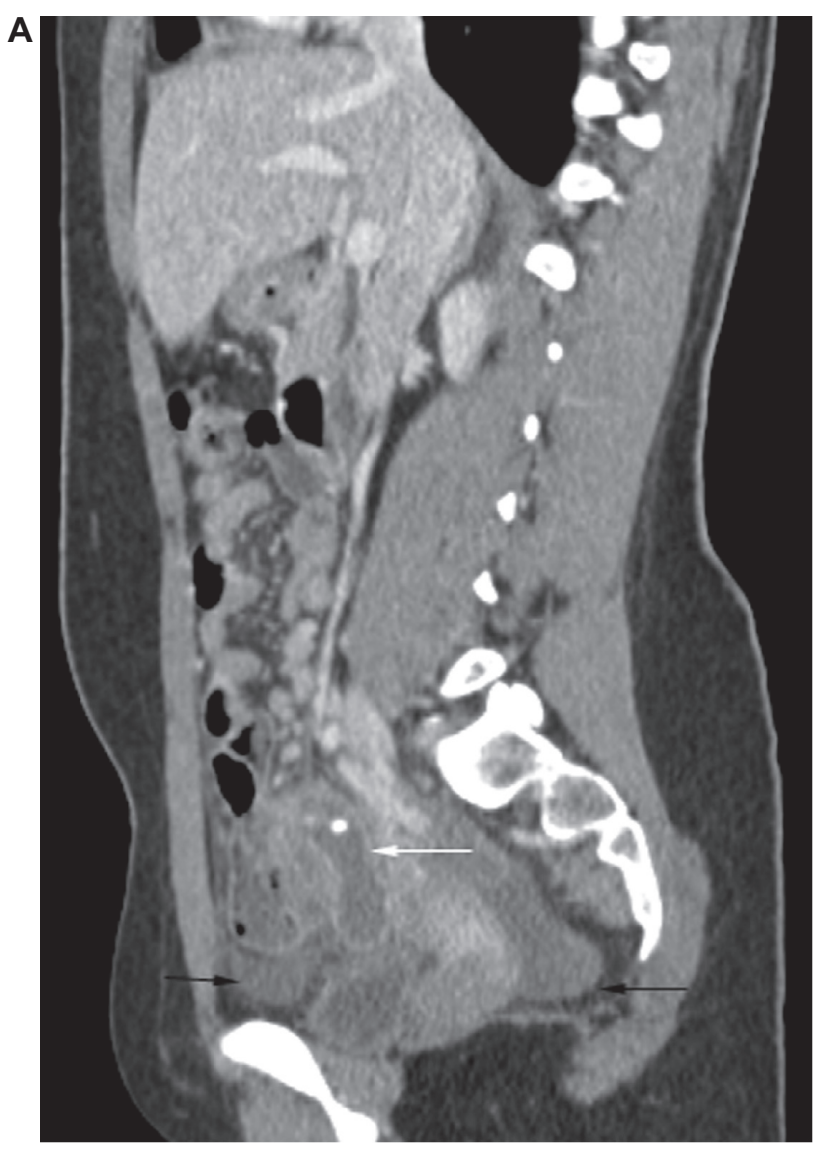

B

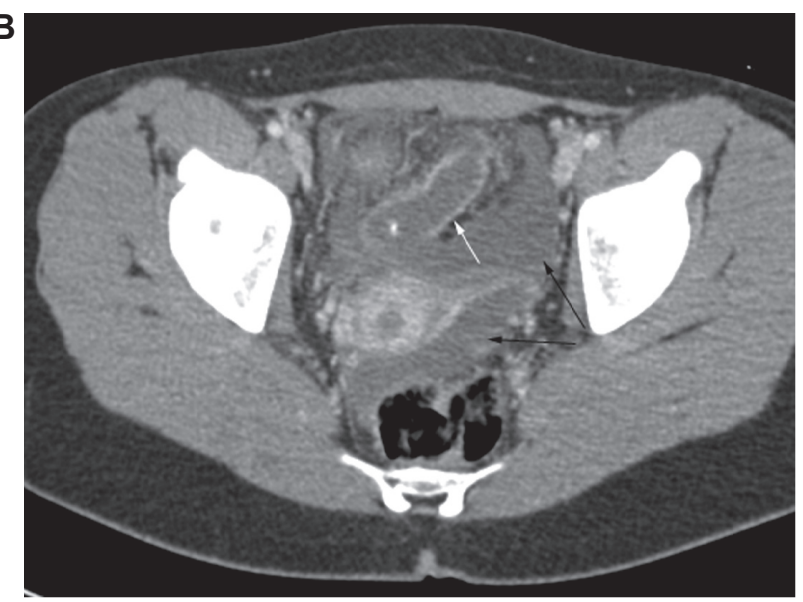

Figure 8 Twelve-year-old girl who presented with a history of right iliac fossa pain. Ultrasound examination was inconclusive. A) and B) Sagittal reformat and an axial image from a CT examination performed following the administration of intravenous contrast medium demonstrating a distended appendix (white arrows) containing an appendicolith. There is adjacent free fluid (black arrows). The findings are in keeping with appendicitis.

cutoff $\operatorname{sign}^{36}$ may be seen on plain abdominal radiographs in cases of acute pancreatitis, but, again, there are no specific radiographic features. As a result, there is no evidence to support the use of the plain abdominal radiograph in this context. Ultrasound is the most frequently performed imaging modality used to evaluate the gallbladder and should
A

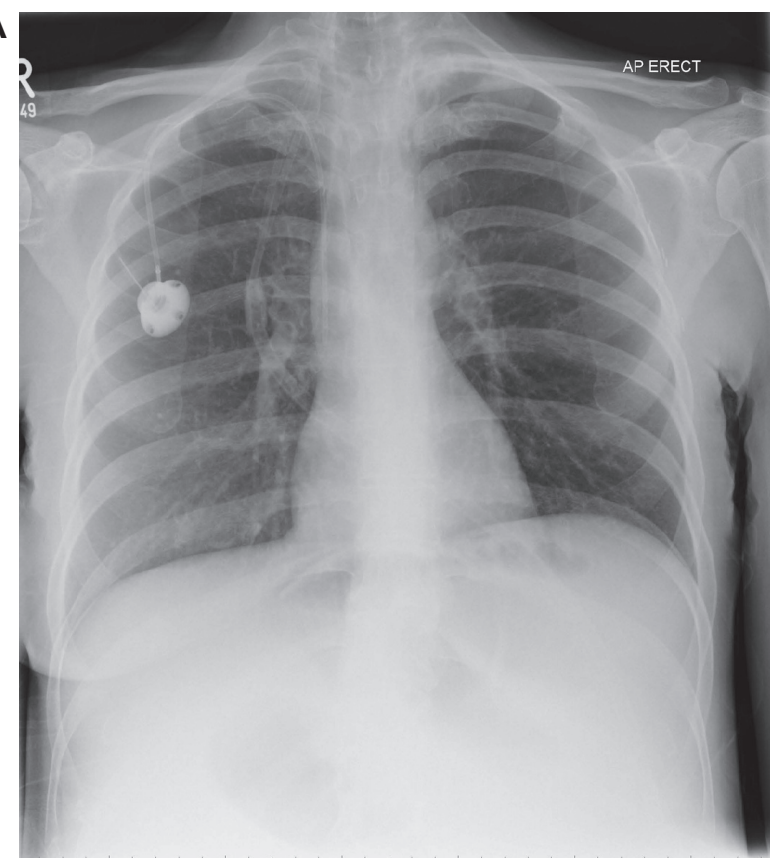

B

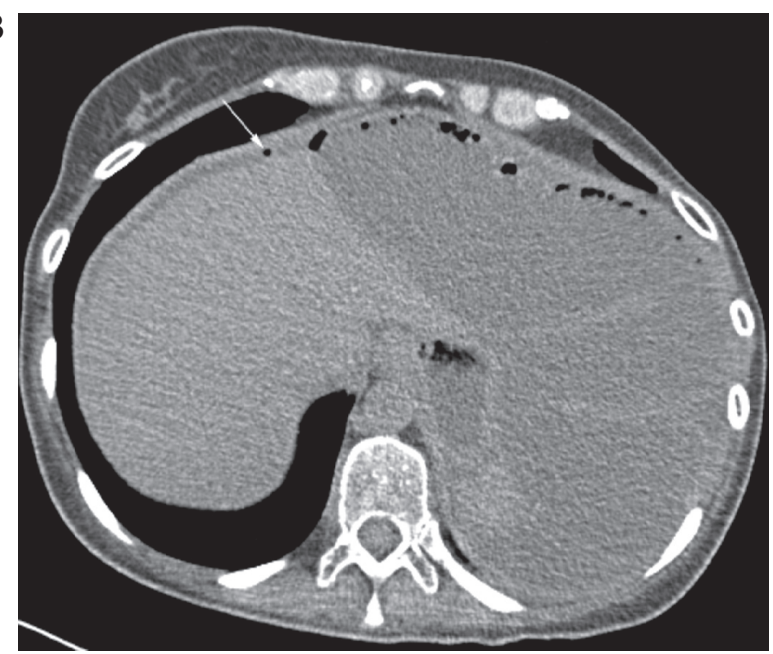

Figure 9 Forty-three-year-old woman with a history of ovarian carcinoma who presented with a history of abdominal pain and vomiting. A) No free air demonstrated under the diaphragm on the erect chest radiograph. B) Axial CT image demonstrating multiple distended loops of obstructed small bowel in the upper abdomen. There were also several small locules of free gas (white arrow) and a small volume of free fluid adjacent to the liver indicating perforation.

be considered the primary imaging technique for patients suspected of having acute cholecystitis. The plain abdominal radiograph has no role here.

\section{Perforated viscus}

There is no evidence to support the use of the plain abdominal radiograph as a tool in the diagnosis of a perforated hollow viscus. The diagnosis of free air is better made with an erect chest radiograph, ${ }^{37}$ and this is currently the first-line radiological investigation. CT is, however, rapidly replacing the erect chest radiograph. It is more sensitive for the detection of 

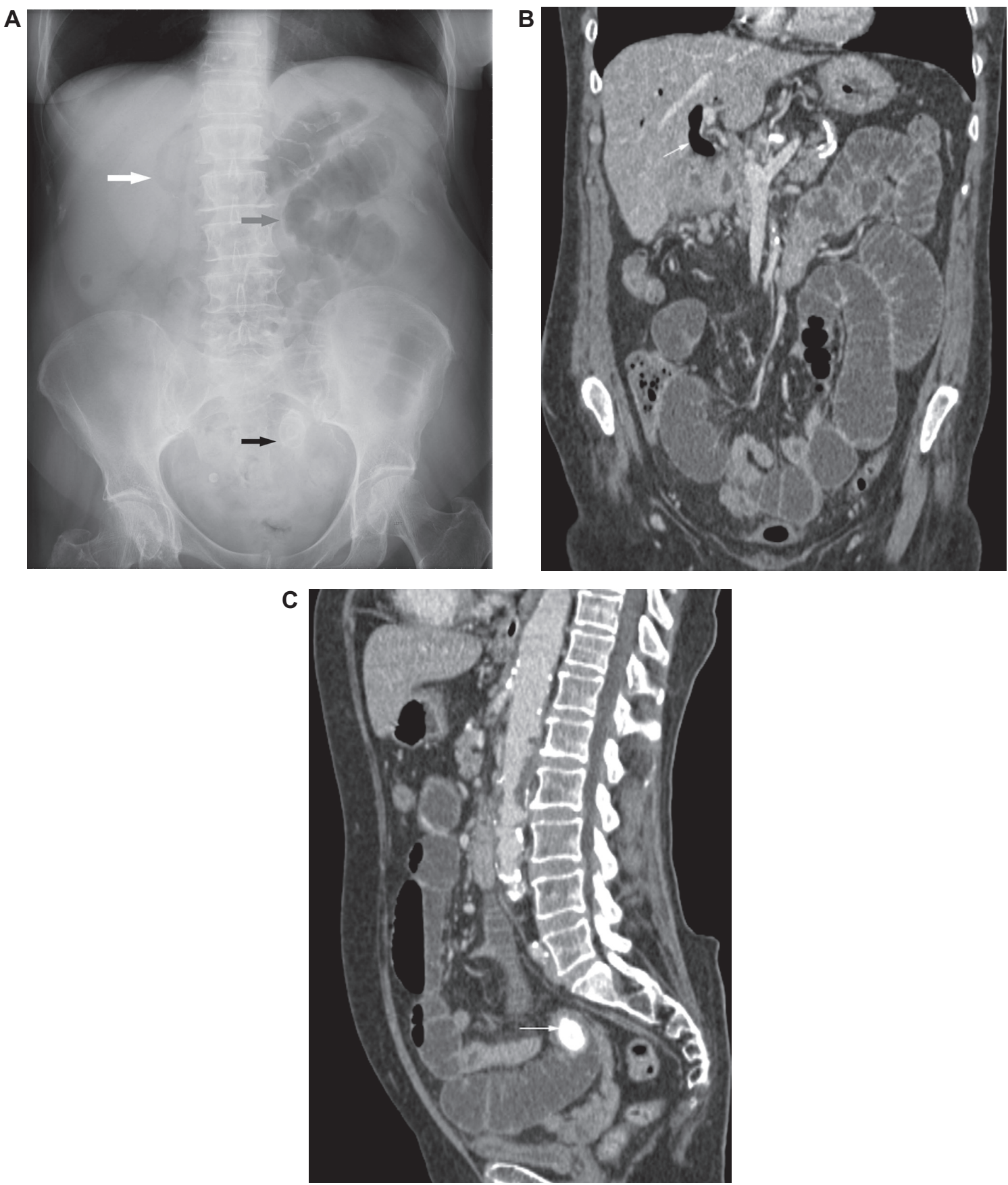

Figure 10 Eighty-three-year-old woman with a history of abdominal pain and vomiting. A) Plain abdominal radiograph demonstrating air in the biliary tree (white arrow), dilated loops of small bowel (grey arrow), and a calcified gallstone projected over the pelvis (black arrow). The findings were in keeping with a gallstone ileus. B) and C) Coronal and sagittal reformats of a CT examination performed after the administration of intravenous contrast medium demonstrating the same findings; air in the biliary tree and SBO caused by a gallstone (white arrow).

small volumes of air $^{38}$ (Figure 9). Another distinct advantage is that it can accurately determine the exact site of perforation in $86 \%$ of cases. ${ }^{39}$ Details relating to the site of perforation and whether it is localized or not are valuable to the surgeon, who may then determine whether conservative management or surgery is required.

\section{Conclusion: the future of the plain abdominal radiograph}

Given the low diagnostic yield of the plain abdominal radiograph in the evaluation of the acute abdomen, it is difficult to understand its continued application. The average plain abdominal radiograph exposes the patient to a typical 


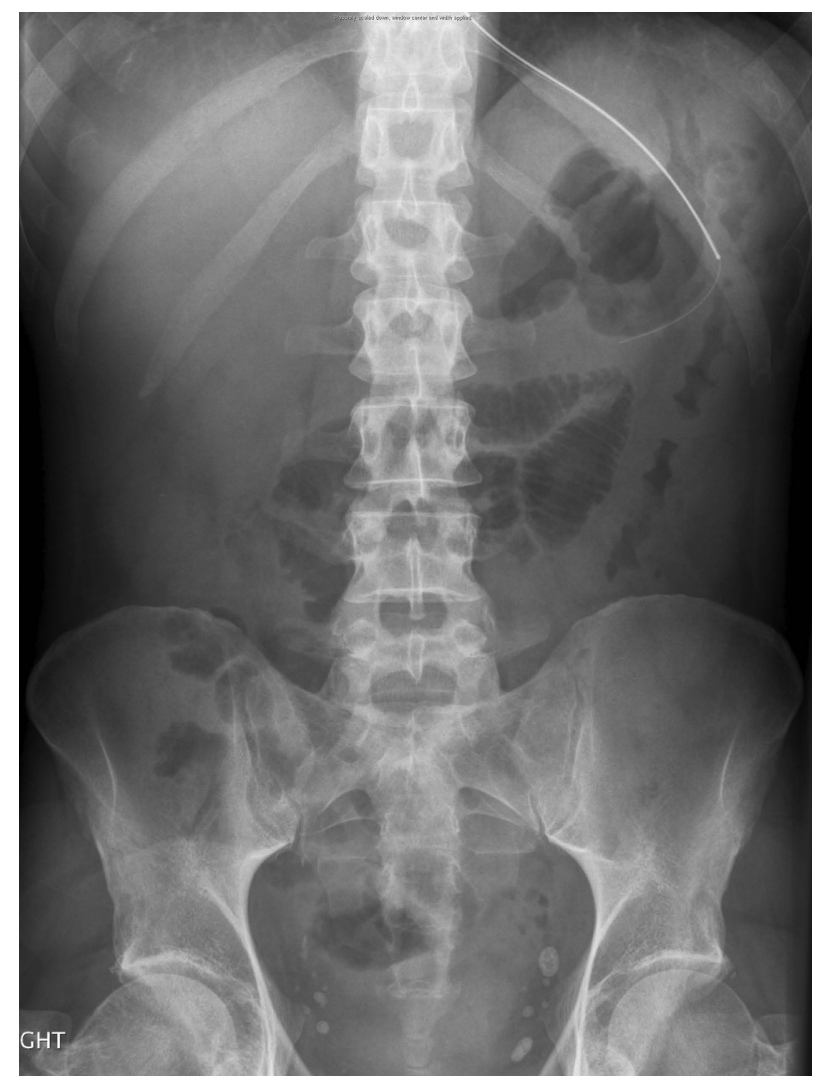

Figure I I Forty-one-year-old man with a history who presented with abdominal pain and bloody diarrhea. The plain abdominal radiograph demonstrated a thick walled descending colon in keeping with colitis.

effective dose of $0.7 \mathrm{mSv}^{11}$ (approximately equivalent to 4 months of natural background radiation), which is equal to 35 chest radiographs. The average abdominal radiograph costs around $£ 20$ to acquire. This does not include the cost of the radiographer performing the examination or the reporting radiologist. ${ }^{40}$ The typical effective dose delivered during a CT examination of the abdomen/pelvis is around $10 \mathrm{mSv} .{ }^{11}$ The average cost of an abdominal/pelvic CT examination is between $£ 400$ and $£ 500$. These figures will vary according to the protocol employed. Although each individual abdominal radiograph is relatively inexpensive, the high volume of investigations performed results in significant costs. If these radiographs are generally unhelpful or even potentially misleading, often necessitating further investigation regardless of the result, this exposure and the associated financial burden are surely unwarranted; other imaging techniques should probably replace them entirely. If abdominal radiography were a new test, introduced today for the investigation of the acute abdomen, it would almost certainly be a failure.
The OPTIMA study group set out to identify the best imaging strategy for the accurate detection of urgent conditions in patients with acute abdominal pain. ${ }^{41}$ They conducted a prospective multicenter study of more than 1000 patients evaluating the added diagnostic value of plain radiographs, ultrasonography, and CT after clinical evaluation. They concluded that although CT is the most sensitive imaging modality for detecting urgent conditions in patients with acute abdominal pain, a strategy that employed CT only after initial radiography and negative/ inconclusive ultrasonography resulted in both the highest overall sensitivity and the minimum radiation exposure. Importantly, the group has now suggested that plain radiography should be entirely excluded from the initial assessment of these patients. ${ }^{18}$

Sala et $\mathrm{al}^{42}$ demonstrated that plain radiography may be avoided if early CT examination is performed. There will, of course, be a price to pay in terms of cost and radiation dose if CT completely replaces plain radiography. These factors seem likely to sustain the use of the plain abdominal radiograph at least in the short term while research into the optimization of imaging strategies continues.

New guidelines are now required to reflect the growing body of evidence against the use of the abdominal radiograph in the context of acute abdominal pain. ED clinicians should surely be encouraged to request more sensitive and specific imaging modalities without delay. The limitations of the abdominal radiograph should be widely publicized, and the factors behind their continued popularity amongst clinicians should be assessed. Do clinicians use abdominal radiographs as a kind of radiological placebo? If so, they may be providing false reassurance. Alternatively, some clinicians may simply be unaware of the potential dangers of overdependence on the abdominal radiograph. Early review by senior clinicians should be encouraged in order that the most appropriate investigations are requested from the outset. This in turn will enable accurate and timely diagnoses to be made, minimizing morbidity and mortality. Although this approach may increase the radiological costs, overall health care costs would decrease.

Until new guidelines are introduced and while the plain abdominal radiograph continues to be utilized in the initial evaluation of the acute abdomen, it is essential that radiologists and emergency clinicians maintain their interpretive skills. Important findings such as pneumoperitoneum, pneumobilia (Figure 10), portal venous gas, intramural gas, bowel wall thickening (Figure 11), SBO, and LBO must be 
reported. We must also insist on high-quality images. The whole abdomen including the hemidiaphragms and the hernia orifices must be visualized.

Radiologists must also report plain abdominal radiographs in a constructive way. It is best to avoid the term 'nonspecific bowel gas pattern' as this encompasses a broad range of appearances and meanings. It is a somewhat confusing expression used to describe a gas pattern that does not fulfil the criteria of more specific diagnoses. At one extreme, it may describe a normal condition, while at the other end of the spectrum, it may reflect bowel obstruction. If there is a potential abnormality, this should be described and correlated with the clinical picture in order that any additional investigations may be carried out without delay.

\section{Disclosure}

The authors report no conflicts of interest in this work.

\section{References}

1. de Dombal FT. Diagnosis of Acute Abdominal Pain. 2nd ed. New York: Churchill Livingstone; 1991:1-10.

2. Schurink GW, Bode PJ, van Luijt PA, van Vugt AB. The value of physical examination in the diagnosis of patients with blunt abdominal trauma: a retrospective study. Injury. 1997;28(4):261-265.

3. Ferrera PC, Verdile VP, Bartfield JM, Snyder HS, Salluzzo RF. Injuries distracting from intra-abdominal injuries after blunt trauma. Am J Emerg Med. 1998;16(2):145-149.

4. Levine MS. Plain film diagnosis of the acute abdomen. Emerg Med Clin North Am. 1985;3(3):541-562.

5. McCook TA, Ravin CE, Rice RP. Abdominal radiography in the emergency department: a prospective analysis. Ann Emerg Med. 1982;11(1):7-8.

6. Gore RM, Miller FH, Pereles FS, Yaghmai V, Berlin JW. Helical CT in the evaluation of the acute abdomen. AJR Am J Roentgenol. 2000; 174(4):901-913.

7. Kundra V, Silverman PM. Impact of multislice CT on imaging of acute abdominal disease. Radiol Clin North Am. 2003;41(6):1083-1093.

8. Leschka S, Alkadhi H, Wildermuth S, Marincek B. Multi-detector computed tomography of acute abdomen. Eur Radiol. 2005;15(12): 2435-2447.

9. Rydberg J, Buckwalter KA, Caldemeyer KS, et al. Multisection CT: scanning techniques and clinical applications. Radiographics. 2000;20(6):1787-1806.

10. Stoker J, van Randen A, Laméris W, Boermeester MA. Imaging patients with acute abdominal pain. Radiology. 2009;253(1):31-46.

11. The Royal College of Radiologists. Making the Best Use of Clinical Radiology Services: Referral Guidelines. London: The Royal College of Radiologists; 2007.

12. American College of Radiology. American College of Radiology practice guidelines for the performance of abdominal radiography. In: American College of Radiology Practice Guidelines. Reston (VA): American College of Radiology; 2006:301-305.

13. Smith JE, Hall EJ. The use of plain abdominal X-rays in the emergency department. Emerg Med J. 2009;26(3):160-163.

14. Markus JB, Somers S, Franic SE, Moola C, Stevenson GW. Interobserver variation in the interpretation of abdominal radiographs. Radiology. 1989;171(1):69-71.

15. Ahn SH, Mayo-Smith WW, Murphy BL, Reinert SE, Cronan JJ. Acute nontraumatic abdominal pain in adult patients: abdominal radiography compared with CT evaluation. Radiology. 2002;225(1): 159-164.
16. MacKersie AB, Lane MJ, Gerhardt RT, et al. Nontraumatic acute abdominal pain: Unenhanced helical CT compared with three-view acute abdominal series. Radiology. 2005;237(1):114-122.

17. Kellow ZS, MacInnes M, Kurzencwyg D, et al. The role of abdominal radiography in the evaluation of the nontrauma emergency patient. Radiology. 2008;248(3):887-893.

18. van Randen A, Laméris W, Luitse JS, et al. The role of plain radiographs in patients with acute abdominal pain at the ED. Am J Emerg Med. 2010 Apr 23; article in press, available on line. doi: 10.1016/j. ajem.2009.12.020.

19. Bohner H, Yang Q, Franke C, Verreet PR, Ohmann C. Simple data from history and physical examination help to exclude bowel obstruction and to avoid radiographic studies in patients with acute abdominal pain. Eur J Surg. 1998;164(10):777-784.

20. Suri S, Gupta S, Sudhakar PJ, Venkataramu NK, Sood B, Wig JD. Comparative evaluation of plain films, ultrasound and CT in the diagnosis of intestinal obstruction. Acta Radiol. 1999;40(4):422-428.

21. Maglinte DD, Reyes BL, Harmon BH, et al. Reliability and role of plain film radiography and $\mathrm{CT}$ in the diagnosis of small-bowel obstruction. AJR Am J Roentgenol. 1996;167(6):1451-1455.

22. Frager D, Medwid SW, Baer JW, Mollinelli B, Friedman M. CT of smallbowel obstruction: value in establishing the diagnosis and determining the degree and cause. AJR Am J Roentgenol. 1994;162(1):37-41.

23. Ros PR, Huprich JE. ACR appropriateness criteria on suspected smallbowel obstruction. J Am Coll Radiol. 2006;3(11):838-841.

24. Godfrey EM, Addley HC, Shaw AS. The use of computed tomography in the detection and characterisation of large bowel obstruction. $N Z$ Med J. 2009;122(1305):57-73.

25. Smith RC, Rosenfield AT, Choe KA, et al. Acute flank pain: comparison of non-contrast-enhanced CT and intravenous urography. Radiology. 1995;194(3):789-794.

26. Sommer FG, Jeffrey RB Jr, Rubin GD, et al. Detection of ureteral calculi in patients with suspected renal colic: value of reformatted non contrast helical CT. AJR Am J Roentgenol. 1995;165(3):509-513.

27. Sourtzis S, Thibeau JF, Damry N, Raslan A, Vandendris M, Bellemans M. Radiologic investigation of renal colic: unenhanced helical CT compared to excretory urography. AJR Am J Roentgenol. 1999;172(6):1491-1494.

28. Smith RC, Verga M, McCarthy S, Rosenfield AT. Diagnosis of acute flank pain: value of unenhanced helical CT. AJR Am J Roentgenol. 1996;166(1):97-101.

29. Levine JA, Neitlich J, Verga M, Dalrymple N, Smith RC. Ureteral calculi in patients with flank pain: correlation of plain radiography with unenhanced helical CT. Radiology. 1997;204(1):27-31.

30. Roa PM, Rhea JT, Rao JA, Conn AK. Plain abdominal radiography in clinically suspected appendicitis: diagnostic yield, resource use, and comparison with CT. Am J Emerg Med. 1999;17(4):325-328.

31. Otero HJ, Ondategui-Parra S, Erturk SM, Ochoa RE, Gonzalez-Beicos A, Ros PR. Imaging utilization in the management of appendicitis and its impact on hospital charges. Emerg Radiol. 2008;15(1):23-28.

32. van Randen A, Bipat S, Zwinderman AH, Ubbink DT, Stoker J, Boermeester MA. Acute appendicitis: meta-analysis of diagnostic performance of CT and graded compression US related to prevalence of disease. Radiology. 2008;249(1):97-106.

33. Cobben LP, Groot I, Haans L, Blickman JG, Puylaert J. MRI for clinically suspected appendicitis during pregnancy. AJR Am J Roentgenol. 2004;183(3):671-675.

34. Pedrosa I, Levine D, Eyvazzadeh AD, Siewert B, Ngo L, Rofsky NM. MR imaging evaluation of acute appendicitis in pregnancy. Radiology. 2006;238(3):891-899.

35. Vu L, Ambrose D, Vos P, Tiwari P, Rosengarten M, Wiseman S. Evaluation of MRI for the diagnosis of appendicitis during pregnancy when ultrasound is inconclusive. J Surg Res. 2009;156(1):145-149.

36. Lee PW. The plain X-ray in the acute abdomen: a surgeon's evaluation. Br J Surg. 1976;63(10):763-766.

37. Flak B, Rowley VA. Acute abdomen: plain film utilization and analysis. Can Assoc Radiol J. 1993;44(6):423-428. 
38. Stapakis JC, Thickman D. Diagnosis of pneumoperitoneum: abdominal CT vs upright chest film. J Comput Assist Tomogr. 1992;16(5): 713-716.

39. Hainaux B, Agneessens E, Bertinotti R, et al. Accuracy of MDCT in predicting site of gastrointestinal tract perforation. AJR Am J Roentgenol. 2006;187(5):1179-1183.

40. Morris-Stiff G, Stiff RE, Morris-Stiff H. Abdominal radiograph requesting in the setting of acute abdominal pain: temporal trends and appropriateness of requesting. Ann R Coll Surg Engl. 2006;88(3):270-274.
41. Laméris W, van Randen A, van Es HW, et al. Imaging strategies for detection of urgent conditions in patients with acute abdominal pain: diagnostic accuracy study. BMJ. 2009;338:b2431.

42. Sala E, Watson CJ, Beadsmoore C, et al. A randomized, controlled trial of routine early abdominal computed tomography in patients with non-specific abdominal pain. Clin Radiol. 2007;62(10):961-969.

Reports in Medical Imaging

\section{Publish your work in this journal}

Reports in Medical Imaging is an international, peer-reviewed, open access journal publishing original research, reports, reviews and commentaries on all areas of medical imaging. The manuscript management system is completely online and includes a very quick and fair peer-review system, which is all easy to use.

\section{Dovepress}

Visit http://www.dovepress.com/testimonials.php to read real quotes from published authors. 